\title{
On the regularity of the bilinear term for solutions to the incompressible Navier-Stokes equations
}

Marco Cannone and Fabrice Planchon

Abstract. We derive various estimates for strong solutions to the Navier-Stokes equations in $C\left([0, T), L^{3}\left(\mathbb{R}^{3}\right)\right)$ that allow us to prove some regularity results on the kinematic bilinear term.

\section{Introduction and definitions.}

The Cauchy problem for the Navier-Stokes equations governing the time evolution of the velocity $u(x, t)=\left(u_{1}(x, t), u_{2}(x, t), u_{3}(x, t)\right)$ and the pressure $p(x, t)$ of an incompressible fluid filling all of $\mathbb{R}^{3}$ is described by the system

$$
\begin{cases}\frac{\partial u}{\partial t}=\Delta u-\nabla \cdot(u \otimes u)-\nabla p, & \\ \nabla \cdot u=0, & x \in \mathbb{R}^{3}, t \geq 0 . \\ u(x, 0)=u_{0}(x), & \end{cases}
$$

The existence of local solutions to this system which are strongly continuous in time and take value in Lebesgue spaces $L^{p}\left(\mathbb{R}^{3}\right)$ is a well known 
result for $p \geq 3$ (see [2]). In the critical case, $p=3$, for which solutions of (1) are invariant by rescaling, one can construct strong solutions in a subclass of $C_{t}\left(L^{3}\right)=C\left([0, T), L^{3}\left(\mathbb{R}^{3}\right)\right.$ ) (see [5], [20], [7], [9]), but their uniqueness within the natural class was proved only recently $([6])$. The key tool in obtaining this uniqueness result was the use of the Besov spaces $\dot{B}_{q}^{3 / q-1, \infty}$, for $q<3$. These spaces have been used previously, but mainly with $q \geq 3$, in obtaining global existence results (see [12], $[2],[18]$ ). In addition, it was already noticed in various contexts (see $[2],[17])$ how the bilinear term, which is the difference between the solution and the solution to the linear heat equation (with same initial data), behaves better than the solution itself. We improve these results in the present paper, and show how this gain in regularity is related to the uniqueness problem, the main estimates involved being of the same kind. Moreover, this allows to extend the decay estimates on the gradient of the solution to (1) obtained by T. Kato in [9].

In order to simplify our study let us introduce the projection operator $\mathbb{P}$ on the divergence free vector fields. We remark that $\mathbb{P}$ is a pseudo-differential operator of order 0 which will be continuous on all spaces subsequently used (primarily because it is continuous on all Lebesgue spaces $L^{p}$, for $\left.1<p<\infty\right)$.

A common method solving (1) is to reduce the system to an integral equation,

$$
u(x, t)=S(t) u_{0}(x)-\int_{0}^{t} \mathbb{P} S(t-s) \nabla \cdot(u \otimes u)(x, s) d s
$$

where $S(t)=e^{t \Delta}$ is the heat kernel, and then to solve it via a fixed point argument in a suitable Banach space (see [2], [9], [10]). Following [2], we remark that the bilinear term in (2) can be reduced to a scalar operator,

$$
B(f, g)=\int_{0}^{t} \frac{1}{(t-s)^{2}} G\left(\frac{\cdot}{\sqrt{t-s}}\right) *(f g) d s,
$$

where $G$ is analytic, such that

$$
\begin{gathered}
|G(x)| \leq \frac{C}{1+|x|^{4}}, \\
|\nabla G(x)| \leq \frac{C}{1+|x|^{4}} .
\end{gathered}
$$


This can be derived easily from the study of the operator under the integral sum, $\mathbb{P} S(t-s) \nabla \cdot$, since its symbol consists of terms like

$$
-\frac{\xi_{j} \xi_{k} \xi_{l}}{|\xi|^{2}} e^{-(t-s)|\xi|^{2}}
$$

outside the diagonal, with another term $\xi_{j} e^{-(t-s)|\xi|^{2}}$ on it. For the sake of simplicity, we will take $G$ as the inverse Fourier transform of $|\xi| e^{-|\xi|^{2}}$.

As we mentioned previously, Besov spaces are a useful tool in studying the bilinear operator $B$. In what follows we will use spaces of functions on $\mathbb{R}^{3}$, so henceforth the reference to the domain space will be omitted. Let us recall the following definition. The reader will find equivalent definitions of Besov spaces in [1], [16], [19].

Definition 1. Let $\theta(x) \in C^{\infty}$ be such that

$$
\hat{\theta}(\xi)=|\xi| e^{-|\xi|^{2}}
$$

Let $p, q \in(1,+\infty), s \in \mathbb{R}, s<1$. Then, $f \in \dot{B}_{p}^{s, q}$ if and only if

$$
\left(\int_{0}^{\infty}\left\|t^{-s} \theta_{t} * f\right\|_{L^{p}}^{q} \frac{d t}{t}\right)^{1 / q}<+\infty
$$

where $\theta_{t}$ is the rescaled function $\theta(\cdot / t) / t^{3}$, and this norm is equivalent to the usual dyadic norm.

We will also make use of the homogeneous version of the SobolevBessel spaces, defined simply by $f \in \dot{H}_{p}^{s}$ if and only if $\Delta^{s / 2} f \in L^{p}$. The reader familiar with Besov spaces will note that by replacing $\hat{\theta}(\xi)$ with $\hat{\theta}(\xi)=|\xi|^{2} e^{-|\xi|^{2}}$ one obtains the usual characterization via the GaussWeierstrass kernel. We will use this fact further in the paper. Among various embeddings between these spaces and the Lebesgue and Sobolev ones, we recall that $L^{p}=\dot{H}_{p}^{0,2}$, for $1<p<\infty$, and $\dot{B}_{2}^{s, 2}=\dot{H}_{2}^{s}=\dot{H}^{s}$, the usual homogeneous Sobolev space.

\section{Theorems and proofs.}

Let us start with the aforementioned result on the regularity of the solution. 
Theorem 1. Let $u(x, t)$ be a solution of $(2)$ in $C\left([0, T), L^{3}\right)$, with initial data $u_{0} \in L^{3}$ and denote by $w$ the function $w=u-S(t) u_{0}$, then

$$
w \in C\left([0, T), \dot{H}_{3 / 2}^{1}\right) .
$$

In other words, the gradient of $w$ is continuous in time with value in $L^{3 / 2}$.

Of course, this estimate makes sense, for, via Sobolev embedding, $\dot{H}_{3 / 2}^{1} \hookrightarrow L^{3}$. This regularity result can be seen in connection with an estimate derived by T. Kato in [9] that assures that $u(x, t)$, the solution of Theorem 1, is such that $t^{1-3 / 2 q} \nabla u(x, t) \in L^{q}$, for $q \geq 3$. Therefore the estimate (8) extends this last estimate to the value $q \geq 3 / 2$ for the bilinear term alone, as if $u_{0}$ only belongs to $L^{3}$, the tendency $S(t) u_{0}$ in general doesn't verify (8).

Let's postpone the proof of the theorem for a moment, and comment further on the meaning of this result. In [17], it was shown that for self-similar solutions (for which the initial data wasn't in $L^{3}$, but in some $\dot{B}_{q}^{-(1-3 / q), \infty}$ with $\left.q>3\right)$, the bilinear term was in $\dot{B}_{3 / 2}^{1,2}$, and it is a simple matter to obtain $\dot{H}_{3 / 2}^{1}$ instead. This is slightly better, as $\dot{H}_{p}^{s} \hookrightarrow \dot{B}_{p}^{s, 2}$ for $p<2$. Now, in order to obtain this result, one makes use of the special structure of a self-similar solution. For such solutions, the time regularity is intimately related to the space regularity because of the scaling $u(x, t)=(1 / \sqrt{t}) U(x / \sqrt{t})$. On the other hand, using $L_{t}^{p}\left(L_{x}^{q}\right)$ estimates, it was proved in [17] that for a solution in $C_{t}\left(L^{3}\right)$ with initial data $u_{0} \in L^{3}$ the function $w \in \dot{B}_{3}^{0,2} \hookrightarrow L^{3}$. One remarks that $\dot{H}_{3 / 2}^{1} \hookrightarrow \dot{B}_{3}^{0,2}$. The proof of that result was a consequence of the following proposition applied with $q=6$.

Proposition 1. Let $3 \leq q \leq 6$. Then the bilinear operator $B(f, g)$ is bicontinuous from

$$
L_{t}^{2 /(1-3 / q)}\left(L_{x}^{q}\right) \times L_{t}^{2 /(1-3 / q)}\left(L_{x}^{q}\right)
$$

into

$$
L_{t}^{\infty}\left(\dot{B}_{q / 2}^{6 / q-1, q /(q-3)}\right)
$$

In particular, if $q=3, B(f, g)$ is bicontinuous from $L_{t}^{\infty}\left(L_{x}^{3}\right) \times$ $L_{t}^{\infty}\left(L_{x}^{3}\right)$ into $L_{t}^{\infty}\left(\dot{B}_{3 / 2}^{1, \infty}\right)$. This last estimate for $q=3$ was used in [6]. 
The proof we are giving here for $3 \leq q \leq 6$ is nothing but paraphrasing the case $q=6$ dealt with in [17]. More precisely, we will prove the estimate by duality. To this end, let $0<T<\infty$. By hypothesis,

$$
\int_{0}^{T}\|f g(x, t)\|_{q / 2}^{1 /(1-3 / q)} d t<\infty
$$

where the integral in time is replaced by a $\sup _{t}=\sup _{t \in[0, T]}$ if $q=$ 3. Then for an arbitrary test function $\varphi(x) \in C_{0}^{\infty}$, we consider the functional

$$
I_{t}=\langle B(f, g), \varphi\rangle
$$

We find

$$
\begin{aligned}
I_{t} & =\int_{0}^{t}\left\langle\frac{1}{(t-s)^{2}} G\left(\frac{\cdot}{\sqrt{t-s}}\right) *(f g), \varphi\right\rangle d s \\
& =2 \int_{0}^{\sqrt{t}}\left\langle f g\left(t-s^{2}\right), \frac{1}{s^{3}} \check{G}\left(\frac{\cdot}{s}\right) * \varphi\right\rangle d s,
\end{aligned}
$$

where $\check{G}(x)=G(-x)$, and we made a change of variable. Applying Hölder inequality both in time and space variables, we get

$$
\begin{aligned}
\left|I_{t}\right| \leq & \left(\int_{0}^{t}\|f g(t-s)\|_{L^{q}}^{1 /(1-3 / q)} d s\right)^{1-3 / q} \\
& \cdot\left(\int_{0}^{\sqrt{t}}\left(\| s^{6 / q-1} \check{G}_{s}(\cdot) * \varphi\right) \|_{q /(q-2)}^{q / 3} \frac{d s}{s}\right)^{3 / q}
\end{aligned}
$$

where $\check{G}_{s}=\check{G}(\cdot / s) / s^{3}$. Using Definition 1 , the second integral is found to be less than the norm of $\varphi$ in $\dot{B}_{q /(q-2)}^{1-6 / q, q / 3}$, which is exactly the dual of $\dot{B}_{q / 2}^{6 / q-1, q /(q-3)}$. The restriction $q \leq 6$ is mainly because we are interested in positive regularity indices. We see that with this proposition we are far from the actual result of Theorem 1, because the third index $p /(p-3)$ is greater than 2. Nevertheless, if we think of uniqueness, we can make a parallel with a recent result, proved (among other things, and in a more general framework) in [8]. We state it here in a pure analytical frame instead of a stochastic one and applying the Besov formalism once again. In order to proceed we need to introduce the following definition involving pseudo-measures. 
Definition 2. A tempered distribution $\psi$ is called a pseudo-measure on $\mathbb{R}^{3}$ if

$$
\sup _{\xi}|\hat{\psi}(\xi)|<\infty
$$

The set of pseudo-measures will be denoted by PM.

Then, we can define Besov type spaces based on pseudo-measures, by replacing the $L^{p}$ norm by the norm on PM in Definition 1 . In the context of the Navier-Stokes equations, this was done before, for example in [12] with Morrey-Campanato spaces. Note that with respect to scaling, the PM norm behaves like an $L^{1}$ norm, so given the scaling invariance of (1) it is natural to introduce the space $\dot{B}_{\mathrm{PM}}^{2, \infty}$, which we define by

$$
\psi \in \dot{B}_{\mathrm{PM}}^{2, \infty} \quad \text { if and only if } \quad \sup _{\xi}|\xi|^{2}|\hat{\psi}(\xi)|<\infty,
$$

whose norm behaves like the $L^{3}$ norm with respect to the scaling invariance. Now we can state:

Theorem 2 ([8]). The bilinear operator $B(f, g)$ is bicontinuous from $L_{t}^{\infty}\left(\dot{B}_{\mathrm{PM}}^{2, \infty}\right) \times L_{t}^{\infty}\left(\dot{B}_{\mathrm{PM}}^{2, \infty}\right)$ into $L_{t}^{\infty}\left(\dot{B}_{\mathrm{PM}}^{2, \infty}\right)$. Therefore there exists a unique global solution to $(2)$ in $L_{t}^{\infty}\left(\dot{B}_{\mathrm{PM}}^{2, \infty}\right)$ provided the initial data is sufficiently small in $\dot{B}_{P M}^{2, \infty}$. Moreover, this solution is self-similar if the initial data is homogeneous of degree -1 .

This result is, to the best of our knowledge, the first where the uniqueness is obtained in the natural space where the initial data is to be taken, instead of in a given subclass. Note that the authors state their theorem within the class of weak solutions, but this assumption is unnecessary in order to obtain Theorem 2. Moreover, the functional class used in [8] obliges to use rather subtle techniques to obtain the continuity of the bilinear operator. However, in the particular case of the pseudo-measures, this is straightforward.

Let us simply see why the bilinear operator is actually continuous. We work in Fourier space, with $\hat{f}$ and $\hat{g}$ instead of $f$ and $g$. A standard argument (rotational invariance and homogeneity) shows that

$$
\frac{1}{|\xi|^{2}} * \frac{1}{|\xi|^{2}}=\frac{C}{|\xi|}
$$


Thus

$$
\mathcal{F}(B(f, g))(t, \xi)=\int_{0}^{t}|\xi| e^{-(t-s)|\xi|^{2}} \hat{f} * \hat{g} d s
$$

and, upon using (16)

$$
\begin{aligned}
\sup _{t, \xi} & \left(|\xi|^{2}|\hat{B}|\right) \\
& \leq C \sup _{t, \xi}\left(|\xi|^{2}|\hat{f}|\right) \sup _{t, \xi}\left(|\xi|^{2}|\hat{g}|\right) \sup _{t, \xi} \int_{0}^{t}|\xi|^{2} e^{-(t-s)|\xi|^{2}} d s .
\end{aligned}
$$

This last integral is in turn less than unity, which concludes the proof once the fixed point algorithm is recalled. The main point here is that we are working within Besov spaces with their third index being equal to $\infty$, and therefore we are allowed to make estimates with the frequency $\xi$ being fixed.

This last statement led the authors in [6] to investigate the spaces $\dot{B}_{q}^{3 / q-1, \infty}$, for $q<3$, and derive the needed estimates to obtain uniqueness. Y. Meyer proved later ([14]) that the bilinear operator is actually bicontinuous on weak $L^{3}$, and gave another way to obtain uniqueness in $L^{3}$. Recall that the bilinear operator is not continuous on $L^{3}$ (see [15] for details). Y. Meyer's estimates are based on a direct characterization of weak $L^{p}$ spaces, and we present here a different proof of this result and obtain a better estimate relating the arguments of both [6] and [14] to [2], [17]. The crucial point here is the embedding, for $q<3$ (see [1])

$$
\dot{B}_{q}^{3 / q-1, \infty} \hookrightarrow L^{3, \infty} .
$$

For the sake of completeness, we add that such a uniqueness result can be derived from earlier estimates on the pressure for the Navier-Stokes system obtained by P. L. Lions ([13]). It should be noted that beyond its usefulness for the uniqueness problem, the forthcoming estimate in itself is yet another variant of the previously quoted regularity result for the non-linear term.

Proposition 2. Let $3 / 2<q<3$. The bilinear operator $B(f, g)$ is bicontinuous from $L_{t}^{\infty}\left(L_{x}^{3, \infty}\right) \times L_{t}^{\infty}\left(L_{x}^{3, \infty}\right)$ into $L_{t}^{\infty}\left(\dot{B}_{q}^{3 / q-1, \infty}\right)$.

Again, we prove the estimate by duality, and fix $q=2$, which gives $1 / 2$ as the regularity index. For an arbitrary test function $\varphi(x) \in C_{0}^{\infty}$, we recall the functional

$$
I_{t}=\langle B(f, g), \varphi\rangle \text {. }
$$


We now have

$$
\begin{aligned}
I_{t} & =\int_{0}^{t}\left\langle\frac{1}{(t-s)^{2}} G\left(\frac{\cdot}{\sqrt{t-s}}\right) *(f g), \varphi\right\rangle d s \\
& =2 \int_{0}^{\sqrt{t}}\left\langle\tilde{G}_{s} * f g\left(t-s^{2}\right), \check{H}_{s}(\cdot) * \varphi\right\rangle d s,
\end{aligned}
$$

where $\check{H}(x)=H(-x), G_{s}=G(\cdot / s) / s^{3}$, and

$$
\hat{G}(\xi)=|\xi| e^{-|\xi|^{2} / 2} e^{-|\xi|^{2} / 2}=\hat{H}(\xi) \hat{\tilde{G}}(\xi)
$$

Then,

$$
\left|I_{t}\right| \leq \int_{0}^{\sqrt{t}}\left\|\tilde{G}_{s} * f g\left(t-s^{2}\right)\right\|_{L^{2}}\left\|\check{H}_{s} * \varphi\right\|_{2} d s .
$$

Using the generalized Young inequality,

$$
\left\|\tilde{G}_{s} f g\left(t-s^{2}\right)\right\|_{L^{2}} \leq C s^{1 / 2}\|f g\|_{L^{3 / 2, \infty}},
$$

we find

$$
\left|I_{t}\right| \leq C \sup _{t}\left(\|g\|_{L^{3, \infty}}\|f\|_{L^{3, \infty}}\right) \int_{0}^{\infty} \sqrt{s}\|\tilde{G} * \varphi\|_{2} \frac{d s}{s}
$$

The last integral is nothing but $\|\varphi\|_{\dot{B}_{2}^{-1 / 2,1}}$, which, by invoking duality, achieves the proof.

One could see Proposition 2 as a counterpart of Theorem 1 for solutions with an initial data in $L^{3, \infty}$. However, we already know that for such solutions, the bilinear term is in $L^{\infty}\left(L^{3}\right)([17])$. This and the aforementioned result on the bilinear term for self-similar solutions tend to indicate we can do better than Proposition 2. We recall here that a solution $u$ to (2) with data in $L^{3, \infty}$ verifies, for all $p>3$

$$
\sup _{t} t^{1 / 2-3 /(2 p)}\|u(x, t)\|_{p}<+\infty
$$

as proved in [18]. This allows to state:

Theorem 3. Let $f(x, t) \in L_{t}^{\infty}\left(L^{3, \infty}\right)$ and $g(x, t)$ verify (23). Then the 
bilinear operator $B(f, g)$ belongs to $L_{t}^{\infty}\left(\dot{H}^{1 / 2}\right)$.

We chose $\dot{H}^{1 / 2}$, but a simple modification of the argument would lead to $\dot{H}_{p}^{3 / q-1}$, for $3 / 2<p<3$. The important point is how the structure of $g$ can be usefully exploited here, and it is indeed such considerations which will lead to the proof of Theorem 1. Before dealing with Theorem 1 , let us first prove Theorem 3 . To this end, let $g$ verify (23) and denote by

$$
\left\|\left.g\right|_{4}=\sup _{t} t^{1 / 8}\right\| g(x, t) \|_{4}
$$

then, by hypothesis

$$
\|\left. g(x, t)\right|_{4} \leq C
$$

We have therefore

$$
\left\|g\left(x, t-s^{2}\right)\right\|_{4} \leq \frac{\|\left. g\right|_{4}}{\left(t-s^{2}\right)^{1 / 8}} .
$$

Upon returning to the estimates of the previous proposition, we obtain, starting from (20)

$$
\left|I_{t}\right| \leq \int_{0}^{\sqrt{t}}\left\|\tilde{G}_{s} * f g\left(t-s^{2}\right)\right\|_{L^{2}}\left\|\check{H}_{s} * \varphi\right\|_{2} d s .
$$

Now we chose different exponents, to get

$$
\left\|\tilde{G}_{s} * f g\left(t-s^{2}\right)\right\|_{L^{2}} \leq C s^{-1 / 4}\|f g\|_{L^{12 / 7, \infty}},
$$

and find

$$
\left|I_{t}\right| \leq C \sup _{t}\left(\left\|\left.g\right|_{4}\right\| f \|_{L^{3, \infty}}\right) \int_{0}^{\sqrt{t}} \frac{\|\tilde{G} * \varphi\|_{2}}{\left(t-s^{2}\right)^{1 / 8} s^{1 / 4}} d s .
$$

Applying Cauchy-Schwarz to this last integral, we bound it from above by

$$
\left(\int_{0}^{1} \frac{d \theta}{\left(1-\theta^{2}\right)^{1 / 4} \theta^{1 / 2}}\right)^{1 / 2}\left(\int_{0}^{\sqrt{t}}\|\tilde{G} * \varphi\|_{2}^{2} d s\right)^{1 / 2} \leq C\|\varphi\|_{\dot{H}^{-1 / 2}}
$$

which concludes the proof of Theorem 3. 
It is worth noting that by combining Proposition 2 and Theorem 3 we can deduce the important uniqueness result in $C_{t}\left(L^{3}\right)$ obtained by Furioli-Lemarié-Terraneo.

Theorem 4 ([6], [14]). Let $u_{0} \in L^{3}$. Then there exists a unique local strong solution of $(2)$ in the class $C_{t}\left(L^{3}\right)$.

The existence of such a solution was proved in [20], [7], [9], where it is also shown that this solution is global if the initial data is small enough (or small enough in a larger space, see [2], [3]). As far as its uniqueness is concerned, consider two solutions $u(x, t)$ and $v(x, t)$ with the same initial data $u_{0}$ and for which $u$ is actually the solution constructed via the fixed point method ([9]). We denote $w=u-S(t) u_{0}$ and $\tilde{w}=v-S(t) u_{0}$. Then, if we temporarily forget that the bilinear operator appearing in (1) is vectorial and non-commutative, we may abuse the notation and write (as in the scalar case)

$$
w-\tilde{w}=2 B\left(S(t) u_{0}, w-\tilde{w}\right)+B(w+\tilde{w}, w-\tilde{w}) .
$$

By applying Theorem 3 to the first term, and Proposition 2 to the second, we obtain

$$
\sup _{t}\|w-\tilde{w}\|_{L^{3, \infty}} \leq C\left(\left\|\left.S(t) u_{0}\right|_{4, T}+\sup _{t}\right\| w+\tilde{w} \|_{3}\right) \sup _{t}\|w-\tilde{w}\|_{L^{3, \infty}}
$$

where $\|\left. f\right|_{4, T}$ indicates we take the $\sup _{t}$ over $(0, T)$ in $(24)$. We then deduce that $w=\tilde{w}$ at least on a small interval in time, as both quantities $\|\left. S(t) u_{0}\right|_{4, T}$ and $\sup _{t}\|w+\tilde{w}\|_{3}$ go to zero when $T$ goes to zero (the first by density and the second by the strong continuity in $L^{3}$ of the solutions). We conclude by a simple continuation argument, as if the time on which the solutions agree were strictly less than the time on which they are defined, we would get a contradiction. And this achieves the proof of Theorem 4. Besides the functional class introduced in [9], other subclasses of $C_{t}\left(L^{3}\right)$ can be used to construct unique solutions as shown for example in [4] where the class arises naturally from the energy inequality.

We are finally in the position to prove Theorem 1. It is useful to rewrite $B$ in a more suitable form, namely

$$
B(f, g)=2 \int_{0}^{\sqrt{t}} G_{s}(x) * f g\left(x, t-s^{2}\right) d s
$$


where, as usual, $G_{s}(x)=G(x / s) / s^{3}$. In addition, it is useful to work with the following operator, $A(f, g)=\Lambda B(f, g)$, where $\Lambda=\sqrt{-\Delta}$ is the Calderón operator, with symbol $|\xi|$. Then

$$
A(f, g)=2 \int_{0}^{\sqrt{t}} \tilde{G}_{s}(x) * f g\left(x, t-s^{2}\right) \frac{d s}{s},
$$

and $\mathcal{F}(\tilde{G})(\xi)=|\xi|^{2} e^{-|\xi|^{2}}$. As we have already noted, if $f$ and $g$ were time independent, then $A$ would reduced to $I-e^{t \Delta}$ applied to the product $f g$. Trying to do this when $f$ and $g$ are two continuous functions of time is bound to fail, and we saw that in fact it leads to Proposition 1. However, when those $f$ and $g$ are coordinates of $u$, the fixed point solution, the construction of $u$ gives various estimates which are very useful as they were in the proof of Theorem 3. In that case we saw that with rather weak $\left(L^{3, \infty}\right)$ initial data, the bilinear term $B(f, g) \in \dot{H}^{1 / 2}$ which represents a substantial improvement in regularity. But if we want to reach the limiting exponents $s=1$ and $p=3 / 2$, we have to be more cautious, and restrict ourselves to initial data in $L^{3}$. Recall the following result ([11]):

Lemma 1. Let $s<1,1<p, q, r<\infty$, such that $1 / p+1 / q=1 / r$. Then

$$
\left\|\Lambda^{s}(f g)\right\|_{r} \leq C\left(\left\|\Lambda^{s} f\right\|_{p}\|g\|_{q}+\left\|\Lambda^{s} g\right\|_{p}\|f\|_{q}\right)
$$

We then will proceed to prove the theorem directly, using this estimate, but to emphasize where the trouble is, we consider

$$
A_{2}=\int_{\sqrt{t / 2}}^{\sqrt{t}} \tilde{G}_{s}(x) * f g\left(x, t-s^{2}\right) \frac{d s}{s} .
$$

This part is easily dealt with, as

$$
\left\|\tilde{G}_{s} * f g\right\|_{3 / 2} \leq\|f g\|_{3 / 2},
$$

which gives

$$
\left\|A_{2}\right\|_{3 / 2} \leq \int_{\sqrt{t / 2}}^{\sqrt{t}} C \sup _{t}\|f g(t)\|_{3 / 2} \frac{d s}{s} \leq C \sup _{t}\|f g(t)\|_{3 / 2} .
$$


But with $A$, we cannot use the effect of the regularizing kernel, as $s$ tends toward 0 . However, let us redefine $A$

$$
A=\int_{0}^{\sqrt{t}} \tilde{\tilde{G}}_{s} *\left(\Lambda^{1 / 2}(f g)\right) \frac{d s}{\sqrt{s}},
$$

where $\hat{\tilde{\tilde{G}}}=|\xi|^{3 / 2} e^{-|\xi|^{2}}$, which allows to move a demi-derivative on $f g$, and lose a factor $\sqrt{s}$. Then, using (29),

$$
\begin{aligned}
\|A\|_{3 / 2} \leq C \int_{0}^{\sqrt{t}} & \left(\left\|\Lambda^{1 / 2} f\left(t-s^{2}\right)\right\|_{3}\left\|g\left(t-s^{2}\right)\right\|_{3}\right. \\
& \left.+\left\|\Lambda^{1 / 2} g\left(t-s^{2}\right)\right\|_{3}\left\|f\left(t-s^{2}\right)\right\|_{3}\right) \frac{d s}{\sqrt{s}} .
\end{aligned}
$$

However, $f$ and $g$ being any coordinates of a solution of (1), they verify the same usual decay estimates as the solutions of the heat equation, namely

Proposition 3 ([9]). Let $p \geq 3$, let $u$ be a solution of (1), then it verifies the estimates,

$$
\mid u \|_{p}=\sup _{t}\left(t^{1 / 2-3 /(2 p)}\|u(x, t)\|_{p}\right)<\infty
$$

and

$$
\mid \nabla u \|_{p}=\sup _{t}\left(t^{1-3 /(2 p)}\|\nabla u(x, t)\|_{p}\right)<\infty .
$$

Therefore,

$$
\left\|\Lambda^{1 / 2} f\left(t-s^{2}\right)\right\|_{3} \leq C \frac{1}{\left(t-s^{2}\right)^{1 / 4}}
$$

Then, from (32), we proceed to obtain

$$
\begin{aligned}
\left\|A_{1}\right\|_{3 / 2} \leq & C \int_{0}^{\sqrt{t}} \frac{d s}{\sqrt{s}\left(t-s^{2}\right)^{1 / 4}} \\
& \cdot \sup _{t}\left(\|f\|_{3}^{3 / 2} t^{1 / 4}\|\Lambda f\|_{3}^{1 / 2}+\|g\|_{3}^{3 / 2} t^{1 / 4}\|\Lambda g\|_{3}^{1 / 2}\right) .
\end{aligned}
$$


As the integral in $s$ is uniformly bounded, this ends the proof, except for continuity. Continuity in zero follows directly from this estimate for $A$, as $t^{1 / 4}\|\Lambda f\|_{3}$ goes to zero as $t$ goes to zero. Continuity elsewhere follows from carefully rewriting $\int_{0}^{t+\varepsilon}-\int_{0}^{t}$ and applying the same argument. It should be added that if we consider the original (vector-valued) operator $B$, instead of the scalar one, based on a Fourier multiplier $|\xi| e^{-|\xi|^{2}}$, we could directly proceed and prove (8) using the estimates of Proposition 3 for $p=3$, as this amounts to deal with $B\left(\partial_{i} f, g\right)$ where $B$ is the generic scalar operator.

As pointed out in [14], we cannot hope to improve the regularity of the bilinear term beyond the space $\dot{B}_{1}^{2,1}$, for the type of initial data we consider here. However, the result of Theorem 1 shows we gain one degree of regularity on the Sobolev embedding line, but as we already mentioned, the limitation comes from the linear part. Therefore, it makes sense to wonder wether we can gain more regularity if we expand the solution $u$ further in terms of the tendency $S(t) u_{0}$. Let us write, with $u_{1}=S(t) u_{0}$

$$
u=u_{1}+B\left(u, u_{1}\right)+B(u, w),
$$

where $B$ is the vector valued operator, and therefore, order matters

$$
B(u, v)=\int_{0}^{t} S(t-s)(u \cdot \nabla) v d s .
$$

Then,

Theorem 5. Let $u_{0} \in L^{3}$, and

$$
u=u_{1}+B\left(u, u_{1}\right)+v
$$

be the solution of $(1)$ which is in $C_{t}\left(L^{3}\right)$. Then

$$
v \in C_{t}\left(\dot{H}_{3 /(2+\alpha)}^{1+\alpha}\right)
$$

with $0<\alpha<1$.

We remark that the limiting value $\alpha$ is not reached. In order to consider this case, it would be necessary to refine the expansion (35) and separate terms coming from the pressure, for which such a limiting case would make sense, from the others. Given the technical complication 
involved, we will avoid this discussion, and prove the simpler result above. It should be also noted that we could split the development of $u$ differently.

The proof works the same way as for Theorem 1. Let us introduce a new operator $A_{\alpha}=\Lambda^{\alpha} \partial_{j} B$

$$
A_{\alpha}(f, g)=2 \int_{0}^{\sqrt{t}} G_{s}^{(\alpha)}(x) *\left(g \partial_{i} f\left(x, t-s^{2}\right)\right) \frac{d s}{s^{\alpha}}
$$

with

$$
\hat{G}^{(\alpha)}(\xi)=|\xi|^{\alpha} \xi_{j} e^{-|\xi|^{2} / 2},
$$

and $i, j \in 1,2,3$. We know that $f$ is any coordinate of $w \in C_{t}\left(\dot{H}_{3 / 2}^{1}\right)$ and $g$ is a coordinate of $u$. In order to simplify the notation, we will use $w, u_{1}, u$ as substitutes for any such coordinate. Then,

$$
\begin{aligned}
\left\|A_{\alpha}(w, u)\right\|_{3 /(2+\alpha)} & \leq C \int_{0}^{\sqrt{t}}\left\|\partial_{i} w\right\|_{3 /(1+\alpha)}\|u\|_{3} \frac{d s}{s^{\alpha}} \\
& \leq C \int_{0}^{\sqrt{t}} \frac{d s}{\left(t-s^{2}\right)^{(1-\alpha) / 2} s^{\alpha}}\left\|\left.\nabla w\right|_{3 /(1+\alpha)} \sup _{t}\right\| u \|_{3},
\end{aligned}
$$

using the decay estimate (34). The integral is bounded independently of $t$, therefore we get $A_{\alpha}(w, u) \in L_{t}^{\infty}\left(L^{3 /(2+\alpha)}\right)$. Continuity can be treated as for Theorem 1.

Acknowledgements. M. Cannone would like to thank A. S. Sznitman for showing him a preprint of his work [8] during a one week visit at the ETH in Zurich, where this paper was initiated. He also benefited from a fruitful discussion with G. Kerkyacharian in Paris. Finally, M. Cannone is grateful for the support from the "Exchange Program between the University of Paris VII and Kyoto University" and for the hospitality he received at the Graduate School of Human and Environmental Studies of Kyoto, where this work was completed.

\section{References.}

[1] Bergh, J., Löfstrom, J., Interpolation Spaces, An Introduction. SpringerVerlag, 1976. 
[2] Cannone, M., Ondelettes, Paraproduits et Navier-Stokes. Diderot Editeurs, 1995.

[3] Cannone, M., Planchon, F., Self-similar solutions for Navier-Stokes equations in $\mathbb{R}^{3}$. Comm. Partial Diff. Equations 21 (1996), 179-193.

[4] Chemin, J. Y., Sur l'unicité pour l'équation de Navier-Stokes tridimensionnelle. In Séminaire X-EDP. Centre de Mathématiques, Ecole Polytechnique, 1996-1997.

[5] Fujita, H., Kato, T., On the Navier-Stokes initial value problem I. Arch. Rational Mech. Anal. 16 (1964), 269-315.

[6] Furioli, G., Lemarié-Rieusset, P. G., Terraneo, E., Sur l'unicité dans $L^{3}\left(\mathbb{R}^{3}\right)$ des solutions mild des équations de Navier-Stokes. C. R. Acad. Sci. Paris 325 (1997), 1253-1256.

[7] Giga, Y., Miyakawa, T., Solutions in $L^{r}$ of the Navier-Stokes initial value problem. Arch. Rational Mech. Anal. 89 (1985), 267-281.

[8] Le Jan, Y., Sznitman, A. S., Cascades aléatoires et équations de NavierStokes. C. R. Acad. Sci. Paris 324 (1997), 823-826.

[9] Kato, T., Strong $L^{p}$ solutions of the Navier-Stokes equations in $\mathbb{R}^{m}$ with applications to weak solutions. Math. Z. 187 (1984), 471-480.

[10] Kato, T., Fujita, H., On the non-stationary Navier-Stokes system. Rend. Sem. Math. Univ. Padova 32 (1962), 243-260.

[11] Kato, T., Ponce, G., Commutator estimates and the Euler and NavierStokes equations. Comm. Pure Appl. Math. XLI (1988), 891-907.

[12] Kozono, H., Yamazaki, M., Semilinear heat equations and the NavierStokes equation with distributions as initial datas. Comm. Partial Diff. Equations 19 (1994), 959-1014.

[13] Lions, P. L., Mathematical topics in fluid mechanics. Volume 1. Oxford University Press, 1996.

[14] Meyer, Y., Wavelets, paraproducts and Navier-Stokes equations. Current developments in Mathematics, International Press (1996), 105-212. To appear.

[15] Oru, F., Rôle des oscillations dans quelques problèmes d'analyse nonlinéaire. Ph. D. Thesis, ENS Cachan, 1998.

[16] Peetre, J., New thoughts on Besov Spaces. Duke Univ. Math. Series. Duke University, 1976.

[17] Planchon, F., Solutions globales et comportement asymptotique pour les équations de Navier-Stokes. Ph. D. Thesis, Ecole Polytechnique, CMAT, Ecole Polytechnique, 1996.

[18] Planchon, F., Asymptotic behavior of global solutions to the NavierStokes equations. Revista Mat. Iberoamericana 14 (1998), 71-93. 
[19] Triebel, H., Theory of Function Spaces. Monographs in Mathematics 78, Birkhauser, 1983.

[20] Weissler, F., The Navier-Stokes initial value problem in $L^{p}$. Arch. $R a$ tional Mech. Anal. 74 (1980), 219-230.

Recibido: 1 de agosto de 1.997

Revisado: 27 de marzo de 1.998

Marco Cannone

U.F.R. Mathématiques

Université Paris 7

2 place Jussieu

75251 Paris Cedex 05, FRANCE cannone@math.jussieu.fr

and

Fabrice Planchon

Program in Applied and Computational Mathematics

Princeton University

Princeton NJ 08544-1000, U.S.A.

fabrice@math.princeton.edu 